\title{
Can CER Be an Effective Tool for Change in the Development and Assessment of New Drugs and Technologies?
}

\author{
Diana I. Brixner, PhD, RPh, and John B. Watkins, PharmD, MPH, BCPS
}

\begin{abstract}
BACKGROUND: Comparative effectiveness research (CER) has been proposed in the United States as a way to compare new drugs and technologies with established alternatives and determine not just whether a therapy works, but how well it works compared to other options.

OBJECTIVES: To define the current use of CER in the development of new drugs and technologies and explore what is needed for this research approach to reduce or stabilize health care costs in the United States.

SUMMARY: In 2010, the Patient-Centered Outcomes Research Institute (PCORI) was established by the Patient Protection and Affordable Care Act (PPACA) to coordinate federally funded CER and recommend research priorities. Hochman and McCormick's (2010) evaluation of 328 randomized trials, observational studies, and meta-analyses involving medications published between June 2008 and September 2009 in 6 key journals showed that most published research did not fulfill the criteria of CER (defined as comparison to active treatment) and that most study design is driven by FDA requirements rather than the need to develop evidence to facilitate selection of the most effective therapy. Since PPACA provides alternative funding for CER, it could encourage funding more studies to help determine which treatment delivers the best value per unit of investment from clinical, humanistic, and economic perspectives. Manufacturers may avoid CER because it increases product development costs, but a drug proven more effective is more likely to be accepted by formulary committees, increasing the drug's market share, whereas payers may reject or limit use of a new drug that performs less effectively in comparative studies.

CONCLUSIONS: CER may not directly reduce expenditures for drugs and medical technologies. The results may vary widely from case to case; however, despite often significantly higher prices for new drugs, it is important to look beyond product costs to the overall impact on health care costs, including medical cost offsets that may occur through improved health or decreased morbidity. To truly decrease cost and improve quality, cost-effectiveness will have to be integrated into CER with the objective of prioritizing efficient therapies in the real-world health care system. If the methods and output of CER improve, the resulting cost-effectiveness ratios will also be more useful to the payer. CER should ultimately, therefore, be a useful tool to help patients, providers, and decision makers provide the most effective and most cost-effective interventions.
\end{abstract}

J Manag Care Pharm. 2012;18(5):S6-S11

Copyright $\odot 2012$, Academy of Managed Care Pharmacy. All rights reserved.

\section{What is already known about this subject}

- The United States leads the world in health care spending as a proportion of gross domestic product. CER is being put forth as a possible way to stabilize or reduce health care costs.

- Health care reform is a driving force behind the increase in CER, with the U.S. government funding research prioritization, infrastructure, and methodology development.

- In 2009, the Institute of Medicine (IOM) released a priority list of 100 research topics derived from a broad stakeholder-input process to help direct future CER efforts.

- Hochman and McCormick (2010) found that 32\% (104 of 328) of studies involving medications published in 6 of the leading general medicine and internal medicine journals in 16 months through September 2009 met the definition of CER (i.e., involved active comparators).

\section{What this article adds}

- The authors (a) describe the progress and development of CER as a health care reform strategy in the United States since 2009; (b) discuss the definition of CER and the types of therapies it can compare, along with possible reasons why a broad interpretation of CER (comparing a drug to a nondrug intervention, for example) may be less relevant to private and public health care payers for whom such broader investments are not considered as part of the budgeting process; and (c) examine the reasons why, from the payer perspective, cost-effectiveness comparison should be part of CER.

$\mathrm{T}$ he rising cost of medical care in the United States has triggered an urgent need for a more efficient health care system that achieves greater demonstrated value. While the annual cost of health care was $\$ 147$ per person in 1960 , by 2008 it had escalated to $\$ 7,845$ per person per year. ${ }^{1}$ Ten percent of overall health care spending in the United States in 2008 was for prescription drugs, compared with 31\% for hospital expenses and 21\% for physician services. The U.S. Department of Health and Human Services (HHS) projects that overall spending for prescription drugs will increase from $\$ 234.1$ billion in 2008 to $\$ 457.8$ billion in 2019. ${ }^{2}$ The average annual increase in drug spending has been 3\%-9\% per year since 2005, based on 4 key factors: the increased annual average number of prescriptions per person; drug price inflation; increases in the number of new drug approvals, especially those that address previously unmet medical needs; and the growing market share of expensive specialty drugs. ${ }^{2}$

The 2011 report by the Organisation for Economic Co-operation and Development (OECD) shows that the United States, compared with other OECD countries, has the highest spending on health care as a proportion of gross domestic product. $^{3}$ From 1970 through 2009, U.S. health care expenditures increased faster than those in "all other high-income OECD countries," with a 5-fold growth rate, even after taking population growth into account.

While there are numerous reason why health care spending in the United States is higher than in other countries, the fact remains that U.S. health costs are some of the highest, and there is an increasing demand to find ways to reduce or stabilize costs while improving health care. As noted by OECD Secretary-General José Ángel Gurría in an August 2009 announcement, "There are opportunities for all countries to improve the performance of their health care system, and making such improvements does not necessarily require higher spending."4 There is hope that comparative effectiveness research (CER) can be used to help improve the performance of 
the U.S. health care system, ideally at a lower or at least more stable expenditure. CER compares how various effective medical treatments improve health outcomes, with the objective of eliminating ineffective services or giving preference to more effective services. This argument has been used to justify the role of CER in U.S. health care reform.

\section{CER as a Health Care Reform Strategy in the United States}

Recognizing that changes needed to be made, the U.S. government is making extensive funds for CER available, mostly favoring infrastructure and methods development. The American Recovery and Reinvestment Act (ARRA) of 2009 led to the establishment of the Federal Coordinating Council for CER. The council was formed to foster coordination of CER and to recommend priorities for funding. ${ }^{5}$ On March 23, 2010, less than a year after the establishment of the Federal Coordinating Council, the Patient Protection and Affordable Care Act (PPACA) became law, which was then amended on March 30, 2010, by the Health Care and Education Reconciliation Act of 2010 (H.R. 4872). ${ }^{6,7}$

The new law focuses on 4 main areas: controlling health care costs and identifying funding and savings opportunities; ${ }^{2}$ expanding health care coverage for a significantly larger number of U.S. citizens, including access to care for pre-existing conditions at affordable premiums and out-of-pocket costs; ${ }^{2}$ improving health care delivery systems; ${ }^{2}$ and establishing sustainability over the long term.

To reach these goals, payment reform will be required, and emphasis on health care must shift toward quality, efficiency, wellness and prevention. With the new law, considerable net reductions in federal deficits are expected over the next 10 years, resulting from several new taxes, fees on health-related industries and cuts in government spending on health care programs such as Medicare Advantage. ${ }^{8}$ As CER develops there may be enough evidence to consider lifting of current regulations that limit price negotiations by the government.

The PPACA initiated and funded the creation of the PatientCentered Outcomes Research Institute (PCORI), a public/ private entity, to coordinate CER and recommend priorities. PCORI replaced the council and is charged with identifying priorities, establishing an agenda, and carrying out primary CER and systematic reviews of existing and future studies. ${ }^{9,10}$ The research conducted for PCORI will be peer-reviewed and made available to the medical community and the general public. The Agency for Healthcare Research and Quality (AHRQ), a federal agency within the HHS charged with supporting research that helps people make more informed decisions and improves the quality of health care services, ${ }^{11}$ can take proactive steps to disseminate the findings of PCORI to physicians, health care providers, patients, insurance providers, and health care technology vendors. ${ }^{10}$

\section{What Is CER?}

The Federal Coordinating Council defined CER as "the conduct and synthesis of systematic research comparing different interventions and strategies to prevent, diagnose, treat and monitor health conditions. The purpose of this research is to inform patients, providers and decision makers, responding to their expressed needs, about which interventions are most effective for which patients under specific circumstances."12 In other words, CER is any research that helps to identify and monitor the right therapy at the right time for the right patient.

The PPACA (2010, Title VI, Subtitle D) also specified what is covered by CER: in addition to medical care, any other strategies or items being used in the treatment, management, and diagnosis of, or prevention of illness or injury in individuals are under the purview of the CER agenda. ${ }^{13}$

Hence, CER is intended to encompass any type of intervention while still considering the overall health outcome. Health technology assessment (HTA) is the analysis of evidence coming from randomized controlled trials (RCTs) and, only exceptionally, from real-world studies. CER aims to bring RCTs and real-world evidence together into an integral framework of comparative evidence. Such evidence may extend CER beyond drug therapy to include nondrug clinical and nonclinical interventions (e.g., the decision to invest in accident prevention or the reduction of environmental risk). This interpretation may be less relevant to private and public health care payers for whom such broader investments are not considered as part of the budgeting process, but may be of interest to employers, since interventions to improve workplace safety and encourage employee wellness may directly benefit them.

PCORI has introduced another term to the CER lexicon: patient-centered outcomes research (PCOR). At first glance, this may seem very similar to CER, but it differs in emphasis. The PCORI Methodology Committee defines PCOR as research that "helps people make informed health care decisions and allows their voice to be heard in assessing the value of health care options. This research answers patient-focused questions such as: (a) "Given my personal characteristics, conditions and preferences, what should I expect will happen to me?" (b) "What are my options and what are the benefits and harms of those options?" (c) "What can I do to improve the outcomes that are most important to me?" (d) "How can the health care system improve my chances of achieving the outcomes I prefer?"14

PCOR emphasizes patient involvement throughout the research process, so that the concerns, perspectives and values of patients are reflected in the methodology and results. This envisions the patient and their health care provider engaging in a collaborative decision-making process as the customers for whom PCOR information is developed. Even though the information generated by PCORI will likely be useful to payers and policy makers, they are not the focus. Patient-centered information and decision support tools will hopefully improve 
both the outcomes and efficiency of the health care process in the United States. This is important, since most of the future federal funding for CER will likely be directed toward PCOR projects

Kindig et al. (2010) describe CER as a holistic approach of comparing the overall effectiveness and performance of health interventions on target outcomes, with the goal of enabling the stakeholder to make more informed choices. ${ }^{13}$ However, it has not yet been defined how far-reaching the actual use of such a CER approach will be and whether CER will focus only on direct health care intervention or will also be used to test preferences for investments in other areas (such as environment, traffic, or education). Using the example of diabetes, CER could be assessed across a broad range of interventions, such as medications, school educational programs, anti-obesity surgery, and preventative behavioral therapy, that all have an impact on diabetes-related health risks. ${ }^{13}$

While a study comparing several pharmaceutical interventions seems logical and feasible, a comparison between pharmaceutical interventions and educational school programs, for example, would be extremely complex, time-consuming, and not suitable for helping to make a decision on reimbursement at the time of a new product launch for a health insurance plan. Furthermore, the current budget and incentive structures in health plans do not necessarily support comparison across budget silos. Therefore, for a formulary decision maker working within the limits of the pharmacy budget, any comparison outside of the pharmacy budget might be less relevant than comparisons within the budget.

\section{The Institute of Medicine's Research Priorities}

As a part of the ARRA, Congress asked the Institute of Medicine (IOM) to prioritize which research questions should be addressed by CER and funded by ARRA. In its 2009 report, the authoring committee developed a priority list of research topics derived from input from a broad array of stakeholders, including policy makers, academics, researchers, the health care industry, physicians and other health care providers, students, and others in the public and private sectors interested in health policy as well as patients, families, and consumers. ${ }^{15}$ For portfolio criteria, the prioritization process examined research areas, populations to be studied, interventions, and proposed methodologies. Condition-level criteria included prevalence, mortality, morbidity, cost, and variability. Priority topic-level criteria included appropriateness of topic for CER, information gaps and duplication, and gaps in translation. ${ }^{15}$

From 2,606 nominated topics, 1,268 were voted on in a 5 -step voting process, and a final list of 100 top priorities, categorized into 4 priority quartiles, was chosen. ${ }^{16}$ These priorities span a broad range of diseases, interventions and investments. The first quartile of priorities includes comparing the effectiveness of various strategies (such as clinical interventions, selected social interventions and combined clinical and social interventions) to prevent obesity, hypertension, diabetes and heart disease in at-risk populations such as the urban poor and American Indians. The first quartile also includes comparing the effectiveness of dissemination and translation techniques to facilitate the use of CER by patients, clinicians, payers, and others. ${ }^{16}$ While both of these seem highly relevant to the health plan, nondrug approaches, such as the effectiveness of yoga in depression or the co-location of psychologists and physicians in children aged 0-3 years, may not be directly relevant, since most of them are not covered services; however, payers may benefit indirectly if effective use of these techniques reduces the need for medication or other medical treatment.

Of the priorities, 6 include diabetes as a key research area, and 3 include obesity. Both diabetes and obesity also have been identified by the OECD as priorities for improvement in health care in the United States. ${ }^{16}$

\section{How Common Is CER Today?}

With the increasing emphasis on CER, a growing number of studies of this type may be expected. Hochman and McCormick (2010) evaluated all human studies published in the 16-month period between June 2008 and September 2009 in 6 key general and internal medicine journals (New England Journal of Medicine, Lancet, JAMA, Annals of Internal Medicine, BMJ, and Archives of Internal Medicine). ${ }^{17}$ In these studies, either a specific medication or a class of medications was compared with either another medication or a nonpharmacologic therapy (active treatment) or either a placebo or no therapy (an inactive control). ${ }^{17}$ Randomized trials, case-control studies, cohort studies, and meta-analyses were included, while systematic reviews and modeling studies were excluded. ${ }^{17}$ Classification as comparative effectiveness (CE) was made if the study involved existing (rather than novel) medications or compared active therapies (active-comparator studies), and non-CE studies were defined as involving novel therapies or comparison to an inactive control such as a placebo (inactive-comparator studies). ${ }^{17}$ Noninferiority RCT studies were categorized as novel therapy because they are generally done to obtain U.S. Food and Drug Administration (FDA) approval. ${ }^{17}$ From a total of 328 studies reviewed, 104 (32\%) were classified as CE studies and 224 $(68 \%)$ as non-CE studies (187 had only an inactive comparator, 81 included non-FDA-approved medications, and 23 were noninferiority trials). ${ }^{17}$

Of the 104 CE studies, 45 compared 2 or more medications (43\%, 95\% confidence interval $[\mathrm{CI}]=34 \%-53 \%), 11$ compared medications with nonpharmacologic interventions (11\%, 95\% $\mathrm{CI}=5 \%-18 \%), 32$ compared different pharmacologic strategies (31\%, 95\% CI $=22 \%-41 \%$ ), and 16 compared different medication doses, durations or frequencies of treatment, or different medication formulations ( $15 \%, 95 \% \mathrm{CI}=9 \%-24 \%){ }^{17}$

Ninety of the 104 CE studies (87\%, 95\% CI=78\%-92\%) 
were funded jointly or exclusively by noncommercial entities. ${ }^{17}$ The authors addressed the fact that "commercial entities presumably devote much of their research to the development of novel therapies and to funding inactive-comparator studies aimed at expanding indications for their products."17 Although analyses are critical for promoting efficient and effective health care, only $1 \%$ of the non-CE studies and $2 \%$ of the CE studies included cost-effectiveness analysis (which could have been due to editorial preferences of the clinically oriented journals). ${ }^{17}$ Overall, Hochman and McCormick found that about two-thirds of research involving medications that was published in high-impact medical journals over 16 months in 2008-2009 does not fulfill the criteria of CER and that much of the study design is driven by FDA requirements rather than the need for evidence allowing the selection of the most effective therapy. ${ }^{17}$

Bourgeois et al. (2012) studied the prevalence of clinical trials addressing 15 priority research topics from the IOM list of priorities. Trials conducted in the United States between 2007 and 2010 and registered on ClinicalTrials.gov were included. The authors reported the prevalence of CER studies, the nature of comparators used, funding and how these factors impacted study results. ${ }^{18}$

The authors found 1,035 trials that met their selection criteria. An additional 3,384 studies were excluded because they were not conducted in the United States, and 2,124 were excluded as not addressing 1 of the selected topics of interest. Among the different types of interventions studied, drug trials had the largest percentage of studies that met the authors' definition of CER (37.2\%), followed by behavior change studies $(28.6 \%)$, procedural interventions $(15.6 \%)$ and devices (13.8\%). ${ }^{18}$ These results align with those reported by Hochman and McCormick ${ }^{17}$ suggesting that their observations are generalizable beyond those 6 journals. In both cases, the majority of studies did not qualify as CER, and the prevalence of CER studies was even lower for nonpharmacologic interventions. Many of the studies found on ClinicalTrials.gov were placebo controlled, and some did not have a control group at all. ${ }^{18}$ The low prevalence of CER studies found by Bourgeois et al. is noteworthy, given that the topics were drawn from the IOM priority list. Furthermore, a large number of studies $(3,384)$ were excluded because they were not conducted in the United States, and without further explanation of the nature of these studies, their data may be considered CER and may be used as such in U.S. formulary decisions. Clearly, there is a great deal of work yet to be done in developing the body of CER evidence that is needed to facilitate informed patient-centered treatment decisions.

To obtain FDA approval, it is possible to demonstrate efficacy of a new product in studies that compare the new product with a placebo. Other countries (government agencies in Canada, the United Kingdom, Japan, and Australia) require studies of new drugs against clinically relevant active comparators. The findings of these head-to-head comparisons are used by health technology assessment agencies and provided to government authorities in these respective countries to help make treatment recommendations or to set pricing for public insurance programs. ${ }^{19,20}$ However, despite the greater requests for this type of data in other countries, there does not appear to be an increase of CER evidence.

In October 2010, Chokshi et al. published another analysis of the design, results, and ultimate impact of past CER studies on practice. They identified 3 areas of special concern: choice of comparison treatments; study time frame; and "external validity"- that is, the extent to which the study's results can be reliably applied to the population as a whole. ${ }^{20}$ However, it should be noted that compared with all other medical or clinical technologies (medical devices, procedures, diagnostics, care pathways, etc.), drugs come to the market with the most advanced evidence base..$^{20}$

Looking forward, it can be expected that CER data satisfying the need for clinical relevance will be required at the launch of a new product. The considerable investment in CER and the formation of independent institutions for CER will eventually lead to the establishment of quality criteria and guidance, as has been seen with the guidance for registration trials of pharmaceutical products.

Increased requests for CER data in decision making do not guarantee a fast process in achieving such evidence because many different stakeholders are involved and their viewpoints need to be considered to provide broad acceptance and, thus, effectiveness of CER. An early and potentially promising effort to address stakeholder concerns is the Academy of Managed Care Pharmacy/National Pharmaceutical Council/ International Society for Pharmacoeconomics and Outcomes Research (AMCP/NPC/ISPOR) Comparative Effectiveness Research Collaborative Initiative (CER-CI). The goal of CER-CI is to establish a consensus-based set of principles and tools to guide the design and evaluation of nonexperimental studies, including prospective and retrospective observational designs, so that the knowledge gained from these studies can be applied to improve patient health outcomes. ${ }^{21}$

\section{Will CER Reduce or Stabilize Health Care Costs?}

CER compares how effective various medical treatments are at improving health outcomes. But does "effective" include the concept of value for money?

From the payer perspective, cost-effectiveness comparison should be part of CER. By comparing the health outcomes of one diabetes drug with another, it is possible to determine the comparative effectiveness. By comparing the health-related quality of life (HRQoL) of the patients treated with one drug with the HRQoL of patients treated with another, it is possible to examine value from the humanistic perspective. Comparing 
the overall cost consequences of one treatment with another, it is possible to determine the economic perspective of effectiveness. Hence, theoretically, CER could help to answer the question of which treatment delivers the best value per unit of investment from clinical, humanistic, and economic perspectives.

The current framework of CER in the United States makes very limited use of cost data, and the PPACA and the Centers for Medicare \& Medicaid Services (CMS) specifically prohibit use of dollars per quality-adjusted life year as a threshold to determine which treatments are cost-effective or recommended. For payers, however, formulary decisions are business decisions as well as clinical. Payers need to offer a comprehensive and reasonable formulary for their membership within the constraints of a given budget. Therefore, cost is an important part of formulary decision making. Payers need to be aware of budget changes when a new drug is integrated into the formulary and whether the expected improved clinical outcome justifies such a change.

In the face of increasing numbers of people becoming eligible for Medicare Part D coverage, options for cost savings will have to be identified and utilized. While it is currently prohibited under the noninterference provision (Medicare Prescription Drug, Improvement, and Modernization Act [MMA] of 2003) for Medicare to negotiate on prices of prescription drugs, overall drug cost could be reduced by starting to negotiate the price. ${ }^{22}$

In 2007, rules such as the safe-harbor guidelines established by CMS and MMA's requirement that Part D plans cover at least 2 drugs per class were put into effect. ${ }^{23}$ In addition, CMS required at least 1 drug in each subclass and gave special protections to 6 classes of drugs, requiring that "all or substantially all drugs" in those classes be included in the formularies. Therefore, Part D drug-price negotiations over anticonvulsants, antidepressants, antineoplastics, antipsychotics, antiretrovirals and immunosuppressants were effectively eliminated. Part D plans regularly exclude some drugs as part of the normal commercial formulary process with other classes of drugs. ${ }^{22}$ One study found that these 6 protected classes accounted for $16.8 \%$ to $33.2 \%$ of Part D drug costs. ${ }^{24}$ Reversing the rule would decrease prices in these classes by $9 \%$ to $11 \%$, for a projected Part D savings of $\$ 511$ million per year. 22,24 Government regulations can thus create challenges for health plans in managing budgets in the absence of CER.

\section{Conclusions}

The impact of CER on reduced drug expenditures may be limited. In fact, CER may lead to higher costs in some cases because it may support a preference for solutions that are clinically more effective and more expensive.

When assessing the impact of cost, it is important to look beyond drug costs to the overall impact on treatment costs, including cost offsets that may occur through improved health or decreased morbidity. In addition, the cost-effectiveness of a technology strongly depends on the efficiency of using it and may differ between individual sites of care (hospitals, practices, care teams, etc.). For example, the cost of a magnetic resonance imaging (MRI) machine used across several hospitals versus the cost of 1 machine purchased for each hospital would significantly decrease the cost per scan based on efficiency of use, not the cost of the machine itself. If cost became a part of CER, the results of such research could contribute to streamlining health care expenditures. To truly decrease cost and improve quality, cost-effectiveness would have to be integrated into CER with the objective of prioritizing efficient therapies in the real-world health care system. ${ }^{25,26} \mathrm{~A}$ final consideration for CER and cost is that if the methods and output of CER improve, the resulting cost-effectiveness ratios will also be more meaningful to the payer.

CER should ultimately, therefore, be a useful tool to help patients, providers, and decision makers provide the optimal and most cost-effective interventions.

\section{Authors}

DIANA I. BRIXNER, PhD, RPh, is Professor and Chair, Department of Pharmacotherapy, University of Utah, College of Pharmacy, Salt Lake City, Utah. JOHN B. WATKINS, PharmD, MPH, BCPS, is Pharmacy Manager, Formulary Development, Premera Blue Cross, Mountlake Terrace, Washington, and Affiliate Professor, Pharmacy, University of Washington, Seattle, Washington.

AUTHOR CORRESPONDENCE: Diana I. Brixner, PhD, RPh, University of Utah, Department of Pharmacotherapy, $30 \mathrm{~S}$. 2000 E., Ste. 258, Salt Lake City, UT 84112. Tel.: 801.581.3182; E-mail: Diana.brixner@pharm.utah.edu. John B. Watkins, PharmD, MPH, BCPS, Premera Blue Cross, MS 432, 7001 220th St. S.W., Mountlake Terrace, WA 98043-2124, Tel.: 425.918.5146;

E-mail:John.Watkins@premera.com.

\section{DISCLOSURES}

This supplement was sponsored by Novo Nordisk Inc. and prepared by the Millcreek Outcomes Group (Salt Lake City, Utah). Diana Brixner reports receiving payment as a manager of Millcreek Outcomes Group to submit this JMCP supplement and also reports being a research investigator on outcomes studies funded by Novo Nordisk to the University of Utah. She reports receipt of payment for educational programs from Novartis and research grants from Abbott, Bristol-Myers Squibb, and Novartis. She is a member of the International Society of Pharmacoeconomics and Outcomes Research (ISPOR) Vision 2020 task force regarding the appropriate communication of outcomes study results to stakeholders and serves on the Academy of Managed Care Pharmacy (AMCP) eDossier Advisory Committee.

John Watkins reports no compensation for participation in the roundtable discussion or for writing and revising portions of this supplement and reports no financial or other potential conflicts of interest related to the subject of this supplement. He is a member of the AMCP Format Executive Committee including oversight of the AMCP Format for Formulary Submissions, and he is a member of the Research Committee of the Foundation for Managed Care Pharmacy (FMCP). He is a member of the ISPOR HTA Council, North America. 


\section{ACKNOWLEDGEMENTS}

The authors thank Anke-Peggy Holtorf, PhD, MBA, for her assistance in gathering and preparing the data used in this article, and Kelley J. P. Lindberg, BS, for writing assistance in preparation of this manuscript.

\section{REFERENCES}

1. Centers for Medicare and Medicaid Services. National health expenditure data: historical. Available at: https://www.cms.gov/ NationalHealthExpendData/02_NationalHealthAccountsHistorical.asp. Accessed April 30, 2012.

2. The Henry J. Kaiser Family Foundation. Prescription drug trends. Publication \#3057-08. May 2010. Available at: http://www.kff.org/rxdrugs/ upload/3057-08.pdf. Accessed April 30, 2012.

3. Organisation for Economic Co-operation and Development. Health at a glance 2011: OECD indicators. 2011. Available at: http://www.oecd.org/ dataoecd/6/28/49105858.pdf. Accessed April 30, 2012.

4. Organisation for Economic Co-operation and Development. Expensive health care is not always the best health care, says OECD's Health at a Glance. August 12, 2009. Available at: http://www.oecd.org/document/14/ 0,3343,en_2649_37407_44216846_1_1_1_1,00.html. Accessed April 30, 2012.

5. Conway PH. How the Recovery Act's Federal Coordinating Council paved the way for the Patient-Centered Outcomes Research Institute. Health Aff (Millwood). 2010;29(11):1-7.

6. Patient Protection and Affordable Care Act. HR 3590, 111th Cong., 2nd Sess. (2010). Public law no. 111-148, 124 Stat 119. Available at: http://www. gpo.gov/fdsys/pkg/PLAW-11lpubl148/pdf/PLAW-11lpubll48.pdf. Accessed April 30, 2012.

7. Health Care and Education Reconciliation Act of 2010. HR 4872, 11lth Cong., 2nd Sess. (2010). Pub law no. 111-152, 124 Stat 1029. Available at: http://www.govtrack.us/congress/billtext.xpd?bill=hlll-4872. Accessed April 30, 2012.

8. Grier P. Health care reform bill 101: who will pay for reform? Christ Sci Monitor. March 21, 2010. Available at: http://www.csmonitor.com/USA/ Politics/2010/0321/Health-care-reform-bill-101-Who-will-pay-for-reform. Accessed April 30, 2012.

9. Patel K. Health reform's tortuous route to the Patient-Centered Outcomes Research Institute. Health Aff (Millwood). 2010;29(10):1777-82.

10. Association of American Medical Colleges. Summary of patient-centered outcomes research provisions prepared by AAMC Government Relations, March 2010. Available at: http://www.aamc.org/download/131994/data/ pcorsummary04022010.pdf. Accessed April 30, 2012.

11. Agency for Healthcare Research and Quality. AHRQ at a glance. May 2011. Available at: http://www.ahrq.gov/about/ataglance.htm. Accessed April 30,2012

12. Draft definition, prioritization criteria, and strategic framework for public comment. HHS.gov/Recovery. Available at: http://www.hhs.gov/recovery/ programs/cer/draftdefinition.html. Accessed April 30, 2012.

13. Kindig D, Mullahy J. Comparative effectiveness—of what? Evaluating strategies to improve population health. JAMA. 2010;304(8):901-02.
14. PCORI. Patient-centered outcomes research. March 5, 2012. Available at: http://www.pcori.org/patient-centered-outcomes-research/. Accessed April 30, 2012.

15. Committee on Comparative Effectiveness Research Prioritization, Board on Health Care Services, Institute of Medicine of the National Academies. Forward. In: Initial National Priorities for Comparative Effectiveness Research. Washington, DC: National Academies Press; 2009. Available at: http://www. iom.edu/Reports/2009/ComparativeEffectivenessResearchPriorities.aspx. Accessed April 30, 2012.

16. Committee on Comparative Effectiveness Research Prioritization, Board on Health Care Services, Institute of Medicine of the National Academies. Initial National Priorities for Comparative Effectiveness Research. Washington, DC: The National Academies Press; 2009. Available at: http://www.iom.edu/ Reports/2009/ComparativeEffectivenessResearchPriorities.aspx. Accessed April 30, 2012.

17. Hochman M, McCormick D. Characteristics of published comparative effectiveness studies of medications. JAMA. 2010;303(10):951-58.

18. Bourgeois FT, Murthy S, Mandl KD. Comparative effectiveness research: an empirical study of trials registered in ClinicalTrials.gov. PLoS One. 2012;7(1):e28820. Epub 2012 Jan 9. Available at: http://www.ncbi.nlm.nih. gov/pmc/articles/PMC3253780/. Accessed April 30, 2012.

19. Clement FM, Harris A, Li JJ, Yong K, Lee KM, Manns BJ. Using effectiveness and cost-effectiveness to make drug coverage decisions: a comparison of Britain, Australia, and Canada. JAMA. 2009;302(13):1437-43. Available at: http://jama.ama-assn.org/content/302/13/1437.full. Accessed April 30, 2012.

20. Chokshi DA, Avorn J, Kesselheim AS. Designing comparative effectiveness research on prescription drugs: lessons from the clinical trial literature. Health Aff (Millwood). 2010;29(10):1842-48.

21. Comparative Effectiveness Research-Collaborative Initiative (CER$\mathrm{CI}$ ). Advancing appropriate use of comparative evidence to improve patient health outcomes. International Society for Pharmacoeconomics and Outcomes Research. Available at: http://www.ispor.org/TaskForces/ InterpretingORSforHCDecisionMakersTFx.asp. Accessed April 30, 2012.

22. Outterson K, Kesselheim AS. How Medicare could get better prices on prescription drugs. Health Aff (Millwood). 2009;28(5):w832-41.

23. Medicare Modernization Act. 2007 final guidelines-formularies. National Association of State Mental Health Program Directors website. Available at: http://www.nasmhpd.org/general_files/policy/Formulary\%20 Guidance\%20CY\%202007.pdf. Accessed April 30, 2012.

24. Kipp RA, Ko C. Medicare Part D administrator survey: potential cost impacts resulting from CMS guidance on "Special Protections for Six Protected Drug Classifications" and Section 176 of the Medicare Improvements for Patients and Providers Act of 2008 (MIPPA) (P.L. 110275). October 16, 2008. Available at: http://www.amcp.org/WorkArea/ DownloadAsset.aspx?id=9279. Accessed April 30, 2012.

25. Weinstein MC, Skinner JA. Comparative effectiveness and health care spending-implications for reform. N Engl J Med. 2010;362(5):46065. Available at: http://www.nejm.org/doi/full/10.1056/NEJMsb0911104. Accessed April 30, 2012.

26. Garber AM, Sox HC. The role of costs in comparative effectiveness research. Health Aff (Millwood). 2010;29(10):1805-11. 